
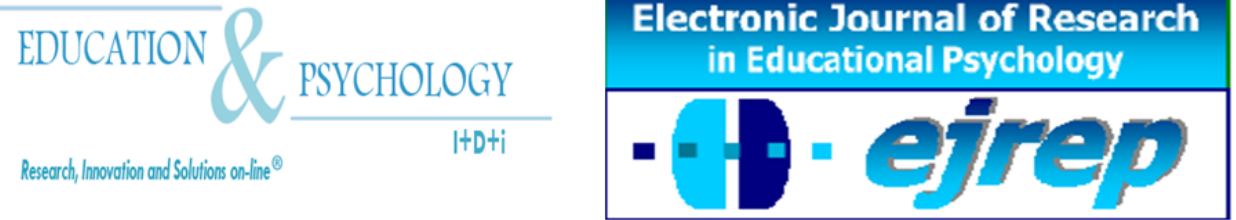

Editorial EOS

\title{
Efectos de un programa de meditación en los valores de una muestra de estudiantes universitarios
}

\section{Clemente Franco Justo ${ }^{1}$, Marisol Navas Luque ${ }^{2}$}

${ }^{1}$ Dpto. de Psicología Evolutiva y de la Educación, Universidad de Almería

${ }^{2}$ Dpto.de Ciencias Humanas y Sociales, Universidad de Almería

\section{España}

Correspondencia: Clemente Franco Justo. Facultad de Ciencias de la Educación. Edificio A. Universidad de Almería. Carretera de Sacramento s/n. La Cañada de San Urbano. Almería 04120. España.

E-mail: cfranco@ual.es

(C) Education \& Psychology $\mathrm{I}+\mathrm{D}+\mathrm{i}$ and Editorial EOS (Spain) 


\title{
Resumen
}

Introducción. Este estudio analiza la influencia de un programa de meditación sobre los valores en una muestra de estudiantes universitarios.

Método. Para ello se contó con la participación de 84 estudiantes universitarios que fueron asignados aleatoriamente a un grupo experimental $(\mathrm{N}=45)$, que recibió la intervención en meditación, y a un grupo control $(\mathrm{N}=39)$, que no fue sometido a dicha intervención. Los valores de los participantes fueron evaluados mediante la versión castellana del Schwartz Value Survey (SVS).

Resultados. Los análisis realizados muestran el incremento de los valores de universalismo, benevolencia, autotrascendencia y colectivismo en el grupo experimental en comparación con el grupo control.

Conclusión. Por tanto, puede concluirse que el proceso de autoconocimiento y exploración interior que caracteriza a la práctica de la meditación, produce una modificación en los valores de aquellos que la practican, produciéndose un incremento de los valores de carácter colectivo y social.

Palabras Clave: meditación, conciencia plena, valores, estudiantes universitarios.

Recibido: 06/03/09 Aceptación inicial: 13/03/09 Aceptación final: 03/09/09

\section{Effects of a meditation program on values in a sample of university students}

\begin{abstract}
Introduction. This study analyzes the influence of a meditation program on the values of a university students' sample.

Method. 84 university students were assigned to an experimental group $(\mathrm{N}=45)$ and a control group $(\mathrm{N}=39)$. The experimental group participates in a meditation program. Values of participants were evaluated with a Spanish adaptation of the Schwartz Value Survey (SVS).

Results. Analysis show an increase of values of universalism, benevolence, transcendence and collectivism in the experimental group, compared with control group.

Conclusion. So, we can conclude that the process of self-knowledge and inner exploration that characterise to meditation produces a change in the values of people practice it, increasing values of collectivism and social type.
\end{abstract}

Keywords: meditation, mindfulness, values, university students.

Received: 03/06/09 Initial Acceptance: 03/13/09 Final Acceptance: 09/03/09 


\section{Introducción}

Desde hace varias décadas el estudio de los valores ha experimentado un creciente interés, debido, en gran medida, a su consideración como principios fundamentales que guían las vidas de los individuos, y también porque la importancia de la educación en valores en nuestra sociedad sigue siendo uno de los retos más importantes en nuesto sistema educativo (De la Fuente, Peralta y Sánchez, 2006). Como señala Cuadrado (2006, p. 585), siguiendo a Schwartz (1992), los valores pueden definirse como "creencias o conceptos que se refieren a conductas o a estados finales deseables, que transcienden una situación específica, que guían la selección o la evaluación de conductas o situaciones, y que están ordenados por importancia relativa". En este sentido, Rokeach (1973, p.19) considera que "un valor es un tipo de creencia, localizada en el centro del sistema total de creencias de una persona acerca de cómo se debe o no se debe comportar, o acerca de algún objetivo de la existencia que vale o no la pena conseguir".

De forma más detallada, González (1990) establece la existencia de cuatro dimensiones diferenciadas respecto a los valores:

a) Son proyectos ideales sobre cómo comportarse y vivir la existencia que la persona aprecia, desea y busca.

b) Son opciones personales que se adquieren como una acción activa de la propia voluntad.

c) Son creencias que se integran en la estructura del conocimiento.

d) Son características de la acción humana que definen la personalidad, orientan la vida y estimulan la conducta.

Desde una perspectiva psicosocial, Schwartz (1992) presenta una teoría integrada sobre el contenido y la estructura de los valores que ha tenido una gran repercusión en la investigación en este ámbito en diferentes países del mundo (una descripción reciente en castellano de esta teoría puede encontrarse en Cuadrado, 2006). La idea central de esta teoría, es que el aspecto fundamental del contenido que diferencia los valores es el tipo de meta motivacional que expresan. Así, en palabras del autor, "los valores representan, en forma de metas conscientes, las respuestas que todos los individuos y sociedades deben dar a tres requisitos universales: las necesidades de las personas como organismos biológicos, los requisitos de la interacción social coordinada, y los requisitos para el correcto funcionamiento y supervivencia de los grupos" (Schwartz, 2001, p.55). De estos tres requisitos se derivan diez tipos moti- 
vacionales de valores, cada uno de ellos compuesto, a su vez, por una serie de valores (véase figura 1) que pueden medirse a través de un cuestionario (Schwartz Values Survey; SVS; Schwartz y Bilsky, 1990).

Poder: Posición y prestigio social, control o dominio sobre personas o recursos (p.e., poder social, reconocimiento social, riqueza).

Logros: Éxito personal como resultado de demostrar competencia según las normas sociales (p.e., ambicioso, capaz, logra éxitos).

Hedonismo: Placer o gratificación sensual para la persona (p.e., placer, disfrutar de la vida). Estimulación: Excitación, novedad y desafíos en la vida (p.e., una vida variada, una vida excitante).

Autodirección: Independencia en la acción, creación y pensamiento (p.e., creatividad, libertad, curioso).

Universalismo: Comprensión, aprecio, tolerancia y protección para el bienestar de toda la gente y para la naturaleza (p.e., igualdad, un mundo en paz, abierto).

Benevolencia: Preocupación por preservar y reforzar el bienestar de personas con las que se tiene contacto personal frecuente (p.e., honesto, leal).

Tradición: Respetar, comprometerse y aceptar las costumbres e ideas que la cultura o la religión imponen a la persona (p.e., respeto a la tradición, humilde).

Conformidad: limitación de las acciones, inclinaciones e impulsos que pueden dañar a otros y violar expectativas o normas sociales (p.e., obediente, autodisciplina)

Seguridad: Seguridad, armonía y estabilidad en la sociedad, en las relaciones interpersonales y en la persona (p.e., seguridad familiar, orden social).

Figura 1. Definición de los 10 tipos motivacionales de valores (Schwartz, 1992)

Schwartz considera que estas motivaciones básicas de valores se organizan, a su vez, en función del interés al que sirven (individualista, colectivista o ambas). Así, las motivaciones de Poder, Logros, Hedonismo, Estimulación y Autodirección sirven al interés de la propia persona (Individualismo); las motivaciones de Conformidad, Tradición y Benevolencia sirven al interés de algún colectivo o grupo social (Colectivismo); y las motivaciones de Universalismo y Seguridad sirven a intereses mixtos (Ambos). Finalmente, Schwartz considera que los tipos de valores se organizan, a su vez, en dos dimensiones bipolares que resumen las oposiciones entre ellos: 
- Autopromoción (Logros y Poder) vs. Autotrascendencia (Universalismo y Benevolencia). Esta dimensión está referida a la preferencia de valores que favorezcan al individuo frente a los que beneficien a la colectividad.

- Apertura al cambio (Autodirección, Estimulación y Hedonismo) vs. Conservación (Tradición, Seguridad y Conformidad). Se trata de la preferencia por los valores abiertos al cambio frente a aquellos que intentan mantener el status quo.

Dada la importancia teórica y empírica que este modelo ha tenido y tiene en la literatura psicosocial, lo utilizaremos en el presente trabajo, así como el cuestionario diseñado por su autor para medir los valores, que será descrito en el apartado de instrumentos.

Creemos que la intervención en el ámbito de los valores, aunque ha demostrado su eficacia a través de programas de intervención psicoeducativa, como por ejemplo el Programa Instruccional-Emotivo para el crecimiento y la Autorrealización personal (PIECAP) (Hernández y Aciego de Mendoza, 1990), no debe limitarse a este formato, sino que puede abrir nuevos campos a la investigación a través de técnicas de naturaleza más introspectiva. De hecho Hernández (1997, 2000, 2002), distingue tres niveles en su modelo de jerarquización de valores, y determina que para llegar al nivel más alto de valores (valores de realización personal), es necesaria una actitud de apertura y expansión hacia determinados aspectos de la realidad, así como desarrollar una actitud de autocontrol y autoconocimiento.

En este sentido, la meditación es una técnica de autoconocimiento y exploración de nuestra realidad interior, que permite alcanzar un estado en el que el cuerpo está relajado y la mente concentrada, lo que permite percibir y ser conscientes de las sensaciones del momento presente mediante una aceptación radical y no valorativa de dicha experiencia (Vallejo, 2006). Este proceso de autoconocimiento y autoexploración, rompe las viejas categorías mentales y los prejuicios, y conduce a la persona que la practica a desvincularse progresivamente de sus pensamientos automáticos, incontrolados e involuntarios (Lager, 2007).

De este modo, el propósito de la meditación es la transformación personal, pues mediante su práctica continuada se va moldeando el carácter mediante un proceso de sensibilización que nos hace más conscientes de nuestros pensamientos y emociones (Holen y Halvor, 2007). Y es que, según considera Campagne (2004), el entrenamiento tanto de la atención como de la des-concentración a través de la práctica de la meditación, desemboca en un ma- 
yor control de las interferencias constantes que hacen de la mente un lugar ruidoso que no permite pensar con claridad, ni distinguir lo esencial de lo irrelevante.

Durante la práctica de la meditación se produce un paso del "modo hacer" al "modo sentir". En el "modo hacer" se dirigen los esfuerzos a conseguir una determinada meta, a vagar entre el pasado y el futuro para reducir la brecha entre cómo son las cosas y cómo nos gustaría que fueran. Por el contrario, en el "modo sentir" se acepta la realidad del momento presente y se expande la conciencia a la experiencia de cada momento, por lo que desaparece cualquier conflicto entre los estados deseados y los reales (Segal, Williams y Teasdale, 2006).

Krishnamurti (1979), establece que la persona "sabia” se observa a sí misma y se conoce a sí misma. Así, el principio de la meditación es el conocimiento propio, tomando conciencia de que la naturaleza de la mente es estar llena de deseos y de pensamientos, para a partir de dicho conocimiento, poder establecer un distanciamiento respecto a los contenidos y los procesos mentales. De esta manera, establece este autor, el ser humano tiene la capacidad de transformarse a sí mismo viendo realmente lo que es, sin que intervenga el proceso analítico del pensamiento. Al mismo tiempo, la meditación provoca el desarrollo de la "atención plena", que puede definirse como una conciencia sin juicios que se desarrolla instante tras instante, momento a momento, mediante un tipo de atención no reactiva, abierta y sin prejuicios en el momento presente (Kabat-Zinn, 2007). Esta toma de conciencia y aceptación de los estados emocionales, puede promover el reconocimiento de los propios valores, el compromiso, la honestidad y las conductas de cooperación, siendo conscientes de los beneficios y de los perjuicios de cómo es la relación que mantenemos con nosotros mismos, con el mundo y con los demás (Iriarte, Alonso-Gancedo y Sobrino, 2006).

En las últimas décadas, son muchos los estudios e investigaciones que han venido comprobando los efectos positivos y favorables de la práctica de la meditación sobre diversos aspectos de la psicología y del comportamiento humano. Por ejemplo, se ha comprobado su eficacia en el tratamiento de la depresión (Carlin y Lee, 1997), la mejora de las habilidades sociales (Beaucheim, Hutchins y Patterson, 2008), el aumento del autoconcepto (Trumbulls y Norris, 1982), la disminución de la ansiedad (Barnes, Bauza y Treiber, 2003), la reducción del estrés (Solberg, Berglund, Ekeberg y Loeb, 1996), el incremento de los niveles de creatividad (Jedrczak, Beresford y Clements, 1985), la reducción del malestar psicológico (MartínAsuero y García de la Banda, 2007), la mejora del bienestar general del sujeto (Westlund, 
1993), la reducción de la percepción del estrés (Franco, 2009a), etc. Sin embargo, el estudio sobre la meditación y su incidencia sobre los valores humanos, ha sido un campo tremendamente ignorado y obviado en la investigación psicológica. Es por ello, que el objetivo de esta investigación es comprobar el impacto que la práctica de la meditación puede producir sobre los valores de un grupo de estudiantes universitarios. Consideramos que, si tal y como se ha planteado a lo largo del marco teórico, las técnicas de meditación provocan un proceso de autoconocimiento y autoexploración interior, necesariamente dicho proceso debe producir un cambio en los valores que guían la vida de aquéllos que la practican.

\section{Método}

\section{Participantes}

Participaron un total de 84 estudiantes de la Universidad de Almería. El grupo experimental estuvo constituido por 45 personas ( 37 mujeres y 8 hombres), y el grupo control lo formaron las 39 personas restantes ( 29 mujeres y 10 hombres). Las edades de los participantes oscilaron entre 18 y 39 años, con una edad media de 22.6 años $($ d.t.=6.4). La asignación de los participantes a uno u otro grupo se realizó aleatoriamente controlándose la variable sexo para que hubiese el mismo número de hombres y mujeres en ambos grupos, y así evitar la interferencia de esta variable en los resultados del estudio.

\section{Instrumentos}

Para medir los valores de los participantes se utilizó la versión castellana validada por Ros y Grad (1991) del Schwartz Value Survey (SVS) (Schwartz y Bilsky, 1990). Este cuestionario consta de 56 valores presentados en dos partes. La primera incluye 30 valores denominados "terminales" (p.e., igualdad, armonía interna, poder social, placer, etc.), y la segunda incluye 26 valores llamados "instrumentales"1 (p.e., independiente, moderado, leal, ambicioso, etc.). Los participantes deben evaluar la importancia que tiene cada valor como principioguía en su vida, utilizando una escala que oscila desde -1 (“Opuesto a mis valores”) hasta 7 ("De suprema importancia").

\footnotetext{
${ }^{1}$ Esta distinción está basada en la propuesta por Rokeach (1973) entre "estados finales" y “modos de conducta".
} 
El cuestionario SVS ha sido validado en más de 60 países, mostrando la cuasiuniversalidad en la estructura de los valores a partir del modelo propuesto por Schwartz (1992). Por esta razón y por la existencia de una versión validada en España con buenos resultados, lo elegimos como instrumento de medida de esta variable (véase Cuadrado, 2006, pp. 596-598 para una comparación con otros cuestionarios de valores).

\section{Diseño}

Para analizar los efectos del programa de meditación (variable independiente) sobre los valores de los estudiantes (variable dependiente), se utilizó un diseño cuasiexperimental de comparación de grupos con medición pretest-postest, con un grupo experimental y un grupo control.

\section{Procedimiento}

En primer lugar se procedió a la obtención de la muestra. Para ello, se ofertó un curso a través del Vicerrectorado de Estudiantes de la Universidad de Almería titulado "Aprendizaje y práctica de la meditación". Al curso se inscribieron un total de 96 estudiantes, de los cuales 84 pasaron a formar parte de la investigación, ya que no se tuvieron en cuenta para los resultados del estudio a aquellos estudiantes que manifestaron haber tenido alguna experiencia con alguna técnica de relajación, yoga, taichi, meditación, etc. De los 84 estudiantes que pasaron a formar parte del estudio, 39 fueron asignados aleatoriamente al grupo control y los 45 restantes al grupo experimental.

Una vez formados los diferentes grupos, se procedió a la evaluación de los valores de los participantes en ambos grupos mediante la aplicación en grupo del SVS en su versión castellana (Ros y Grad, 1991). Posteriormente, se aplicó en el grupo experimental el programa de intervención, el cual consistó en el aprendizaje de una técnica de meditación. Mientras tanto, a los participantes del grupo control se les dijo que con ellos se iniciaría dicho programa más adelante, pues la persona encargada de darles el curso había caído enferma.

El programa de intervención aplicado en el grupo experimental, consistió en el aprendizaje y práctica diaria de una técnica de meditación llamada Meditación Fluir (Franco, 2007, 2009b). El objetivo principal de esta técnica de meditación, no consiste en tratar de controlar 
los pensamientos, sensaciones o emociones, tampoco en modificarlos o cambiarlos por otros, ni en tratar que la mente deje de pensar y de generar pensamientos. Por el contrario, se trata de desarrollar un estado de atención y conciencia plena sobre la actividad mental, aceptando cualquier pensamiento o sensación que pueda aparecer o surgir de forma espontánea, siendo conscientes de la transitoriedad de dicha actividad mental.

La esencia de este tipo de meditación es, por tanto, la de ser conscientes de forma pasiva y sin esfuerzo de lo que ocurre en nuestra mente y en nuestro cuerpo, pero sin tratar de hacer ningún esfuerzo por tratar de cambiarlo o modificarlo, percibiendo las cosas tal y como son y tienen en lugar en cada momento. Durante la práctica de este tipo de meditación, tomamos conciencia de nuestros pensamientos, pero no pensamos sobre ellos, sobre su contenido o veracidad, sino que vamos desarrollando la conciencia de que los pensamientos (al igual que las sensaciones) cambian a cada instante y están fluyendo constantemente.

La práctica de la Meditación Fluir consiste en la repetición mental de un sonido mientras se dirige la atención a la respiración en la zona del abdomen. Ser conscientes de la respiración no quiere decir que tengamos que intervenir sobre ella, sino que simplemente debemos observarla tal y como ocurre y tiene lugar en cada preciso instante, dándonos cuenta de qué estamos respirando, y de cómo estamos respirando. En esta técnica de meditación se utiliza la concentración pasiva donde se dirige la atención de la mente hacia la respiración mientras se repite el sonido, pero si nos distraemos se acepta y se permite dicha distracción para volver a dirigir nuevamente la atención sobre el objeto de meditación.

La práctica de este tipo de meditación, supone retornar una y otra vez la atención hacia el momento presente (la respiración), abriéndonos a la experiencia de cada momento, simplemente siendo conscientes de la realidad del aquí y ahora sin emitir ningún juicio de valor o reacción mental. Es por ello, que la Meditación Fluir nos lleva a comprender a través de la experiencia directa, que los pensamientos surgen y desaparecen continuamente y que están sometidos a un continuo flujo, ya que no hay mejor forma de experimentar el flujo y el cambio de todos los procesos físicos y mentales, que la observación y la toma de conciencia de nuestra respiración, que está surgiendo y cambiando instante tras instante.

El aprendizaje de dicha técnica de meditación es completado con la presentación y discusión de diversas metáforas y ejercicios utilizados en la Terapia de Aceptación y Com- 
promiso (Hayes, Stroshal y Wilson, 1999; Wilson y Luciano, 2002), junto con relatos de la tradición zen (Deshimaru, 2006), y de la meditación vipassana (Hart, 1994). Dichas metáforas, relatos y ejercicios tienen como objetivo igualmente aprender a tomar distancia de los pensamientos, sentimientos y emociones, aprendiendo a observarlos de una manera imparcial, sin dejarnos involucrar o influir por su contenido.

El programa de meditación aplicado al grupo experimental tuvo una duración de 10 semanas, con una periodicidad de una sesión semanal de 90 minutos de duración. Al finalizar el entrenamiento en meditación en este grupo experimental, se procedió a evaluar nuevamente los valores de los participantes en ambos grupos con el SVS para que fuese completado en las mismas condiciones que en la fase prestest. Una vez finalizada la investigación, se procedió a impartir el curso de meditación a los participantes del grupo control.

\section{Resultados}

Los valores fueron agrupados siguiendo el modelo teórico de Schwartz ya descrito en la introducción (ver cuadro 1 y las siguientes agrupaciones). Para analizar la existencia de diferencias estadísticamente significativas entre las medidas pretest y postest tanto en el grupo experimental como en el grupo control, se utilizó la prueba $t$ de Student para muestras relacionadas. Asimismo, se realizó un análisis entre el grupo experimental y el grupo control a partir de las diferencias pretest y postest entre ambos grupos, utilizando para ello la prueba $t$ de Student para muestras independientes. Todos los análisis fueron realizados con el paquete estadístico SPSS versión 15.0. 
Tabla 1. Medias y desviaciones típicas pretest y postest correspondientes a los grupos control y experimental en las agrupaciones de valores del estudio.

\begin{tabular}{|c|c|c|c|c|c|c|c|c|}
\hline \multirow[b]{3}{*}{ Variable } & \multicolumn{4}{|c|}{ PRETEST } & \multicolumn{4}{|c|}{ POSTEST } \\
\hline & \multicolumn{2}{|c|}{ Control } & \multicolumn{2}{|c|}{ Experim. } & \multicolumn{2}{|c|}{ Control } & \multicolumn{2}{|c|}{ Experim. } \\
\hline & $M$ & $D T$ & $M$ & $D T$ & $M$ & $D T$ & $M$ & $D T$ \\
\hline Poder & 2.08 & 0.93 & 1.87 & 1.21 & 2.14 & 0.84 & 1.68 & 1.14 \\
\hline Logros & 4.86 & 1.12 & 4.78 & 1.43 & 4.74 & 1.03 & 4.48 & 1.14 \\
\hline Hedonismo & 4.78 & 1.34 & 4.92 & 1.11 & 4.91 & 1.24 & 4.83 & 1.03 \\
\hline Estimulación & 3.98 & 1.21 & 3.79 & 1.46 & 3.91 & 1.14 & 3.94 & 1.28 \\
\hline Autodirección & 4.58 & 0.96 & 4.79 & 1.03 & 4.71 & 1.03 & 4.86 & 1.09 \\
\hline Universalismo & 4.96 & 1.32 & 5.01 & 0.98 & 4.84 & 1.21 & 5.89 & 0.64 \\
\hline Benevolencia & 4.91 & 0.96 & 4.74 & 0.74 & 4.83 & 0.82 & 5.36 & 0.81 \\
\hline Tradición & 3.45 & 0.67 & 3.62 & 0.87 & 3.54 & 0.73 & 3.49 & 0.79 \\
\hline Conformidad & 4.41 & 1.11 & 4.35 & 1.34 & 4.52 & 1.15 & 4.43 & 1.18 \\
\hline Seguridad & 4.37 & 0.94 & 4.59 & 1.06 & 4.28 & 0.78 & 4.68 & 0.89 \\
\hline Individualismo & 3.72 & 0.63 & 3.98 & 0.78 & 3.64 & 0.71 & 3.79 & 0.82 \\
\hline Colectivismo & 4.34 & 0.97 & 4.21 & 0.85 & 4.27 & 0.82 & 4.83 & 0.93 \\
\hline Ambos & 4.72 & 0.83 & 4.81 & 0.97 & 4.81 & 0.72 & 4.89 & 0.84 \\
\hline Autopromoción & 3.28 & 0.63 & 3.46 & 0.72 & 3.21 & 0.69 & 3.39 & 0.81 \\
\hline Autotrascendencia & 4.69 & 0.94 & 4.91 & 0.74 & 4.74 & 0.82 & 5.59 & 0.87 \\
\hline Apertura al cambio & 4.56 & 0.86 & 4.71 & 0.90 & 4.42 & 0.91 & 4.63 & 0.80 \\
\hline Conservación & 4.13 & 0.97 & 4.21 & 0.82 & 4.26 & 0.76 & 4.30 & 0.91 \\
\hline
\end{tabular}

Diferencias entre el grupo experimental y el control en los valores analizados (pretest y postest)

El análisis estadístico de las diferencias de las puntuaciones pretest en valores entre el grupo control y experimental, mostró la ausencia de diferencias significativas de partida entre ambos grupos en las distintas agrupaciones de valores analizadas (ver Tabla 2). 
Tabla 2. Prueba t de Student para muestras independientes de las diferencias pretest y postest entre el grupo control y experimental en las agrupaciones de valores del estudio.

\begin{tabular}{|c|c|c|c|c|}
\hline \multirow[b]{2}{*}{ Variable } & \multicolumn{2}{|c|}{ PRETEST } & \multicolumn{2}{|c|}{ POSTEST } \\
\hline & $t$ & $p$ & $t$ & $p$ \\
\hline Poder & 1.62 & .148 & 1.45 & .126 \\
\hline Logros & 1.12 & .186 & 0.91 & .234 \\
\hline Hedonismo & 0.31 & .711 & 0.38 & .643 \\
\hline Estimulación & 0.39 & .673 & 0.26 & .628 \\
\hline Autodirección & 0.24 & .693 & 0.17 & .719 \\
\hline Universalismo & 0.81 & .302 & 3.79 & $.001 * *$ \\
\hline Benevolencia & 1.12 & .196 & 4.14 & $.001 * *$ \\
\hline Tradición & 1.34 & .167 & 0.32 & .684 \\
\hline Conformidad & 0.11 & .864 & 0.71 & .348 \\
\hline Seguridad & 0.49 & .506 & 0.31 & .719 \\
\hline Individualismo & 0.81 & .299 & 0.87 & .282 \\
\hline Colectivismo & 0.41 & .485 & 3.84 & $.001 * *$ \\
\hline Ambos & 0.78 & .324 & 0.57 & .414 \\
\hline Autopromoción & 0.43 & .412 & 0.37 & .491 \\
\hline Autotrascendencia & 0.81 & .279 & 5.17 & $.000 *$ \\
\hline Apertura al cambio & 1.03 & .209 & 0.97 & .209 \\
\hline Conservación & 1.45 & .164 & 1.26 & .189 \\
\hline
\end{tabular}

Nota: ${ }^{*} p<.001 ; * * p=.001$

Para comprobar si se habían producido cambios en los valores del grupo experimental respecto al grupo control una vez finalizada la intervención, se realizó un análisis de las diferencias postest entre las medidas de ambos grupos. Los resultados muestran la existencia de diferencias estadísticamente significativas entre ambos grupos en los valores de trascendencia $(t=5.17 ; p<.001)$, benevolencia $(\mathrm{t}=4.14 ; p=.001)$, colectivismo $(t=3.84 ; p=.001)$, y universalismo $(t=3.79 ; p=.001)$. En todos los casos, los participantes que habían realizado el programa de meditación (grupo experimental) mostraban puntuaciones medias más altas en esos valores en comparación con los participantes del grupo control (ver Tabla 2). 
Diferencias entre el pretest y el postest en cada uno de los grupos (experimental y control)

Para analizar si se habían producido cambios en los valores de los participantes de ambos grupos entre el pretest y el postest, se realizó un análisis estadístico mediante la prueba $t$ de Student para muestras relacionadas. Sólo se obtuvieron diferencias estadísticamente significativas entre las puntuaciones medias obtenidas en el pretest y el postest en los valores analizados en el grupo experimental (ver tabla 3). Concretamente, estas diferencias se producen en los valores de trascendencia $(t=5.19 ; p<.001)$, colectivismo $(t=3.93 ; p=.001)$, universalismo $(t=3.89 ; p=.001)$, y benevolencia $(t=3.36 ; p<.005)$, que en todos los casos aumentan sus puntuaciones medias. En el caso del grupo control, no se encontró ninguna diferencia estadísticamente significativa entre las puntuaciones medias en valores en ambas fases del estudio (pretest-postest).

Con el objetivo de poder valorar la magnitud del cambio una vez finalizada la intervención, se empleó la $d$ de Cohen (1988), donde valores superiores a 0.8 indican cambios muy importantes, entre 0.5 y 0.8 importantes, por debajo de 0.5 medios, y menores de 0.2 bajos.

En la Tabla 3 se observa como las puntuaciones de la $d$ de Cohen en el grupo control, muestran la existencia de cambios bajos o muy bajos, con valores que oscilan entre 0.048 y 0.131; mientras que en el grupo experimental alcanza niveles superiores a 1 en benevolencia $(d=1.60)$, y en universalismo $(d=1.07)$, lo que indica que se producen cambios muy importantes en dichas variables tras la aplicación del programa de intervención en meditación. Asimismo, se producen cambios importantes en trascendencia $(d=0.85)$, y en colectivismo $(d=0.696)$. Finalmente, se producen cambios de nivel medio en individualismo $(d=0.240)$, y en logros $(d=0.232)$. 
Tabla 3. Prueba t de Student para muestras relacionadas de las diferencias postest-pretest y d de Cohen en el grupo control y experimental, en las agrupaciones de valores del estudio.

\begin{tabular}{|c|c|c|c|c|c|c|}
\hline \multirow[b]{2}{*}{ Variable } & \multicolumn{3}{|c|}{ Control } & \multicolumn{3}{|c|}{ Experimental } \\
\hline & $t$ & $p$ & $d$ & $t$ & $p$ & $d$ \\
\hline Poder & 0.33 & .744 & 0.04 & 1.32 & .215 & 0.16 \\
\hline Logros & 0.62 & .543 & 0.06 & 1.48 & .292 & 0.23 \\
\hline Hedonismo & 0.97 & .323 & 0.10 & 0.82 & .314 & 0.16 \\
\hline Estimulación & 1.19 & .247 & 0.05 & 1.67 & .268 & 0.10 \\
\hline Autodirección & 1.13 & .263 & 0.13 & 0.42 & .409 & 0.06 \\
\hline Universalismo & 0.25 & .803 & 0.09 & 3.89 & $.001 * *$ & 1.07 \\
\hline Benevolencia & 0.79 & .437 & 0.08 & 3.36 & $.003^{* * *}$ & 1.60 \\
\hline Tradición & 0.20 & .842 & 0.13 & 1.19 & .268 & 0.15 \\
\hline Conformidad & 1.34 & .183 & 0.09 & 0.96 & .318 & 0.06 \\
\hline Seguridad & 0.13 & .893 & 0.10 & 1.23 & .249 & 0.09 \\
\hline Individualismo & 0.21 & .836 & 0.12 & 1.09 & .282 & 0.24 \\
\hline Colectivismo & 0.17 & .878 & 0.07 & 3.93 & $.001 * *$ & 0.69 \\
\hline Ambos & 1.52 & .137 & 0.11 & 0.62 & .384 & 0.08 \\
\hline Autopromoción & 1.38 & .172 & 0.10 & 0.91 & .346 & 0.09 \\
\hline Autotrascendencia & 1.61 & .124 & 0.05 & 5.19 & $.000 *$ & 0.85 \\
\hline Apertura al cambio & 1.34 & .180 & 0.15 & 0.81 & .321 & 0.09 \\
\hline Conservación & 0.91 & .342 & 0.07 & 0.13 & .810 & 0.19 \\
\hline
\end{tabular}

Nota: ${ }^{*} p<.001 ; * * p=.001 ; * * p<.005$

\section{Discusión y Conclusiones}

El objetivo de este estudio era comprobar la influencia de la práctica de la meditación sobre los valores de una muestra de estudiantes universitarios. Para ello, se contó con dos grupos de participantes que presentaban puntuaciones medias similares en todos los valores del cuestionario utilizado (pretest). La hipótesis de partida era que el programa de meditación aplicado (Meditación Fluir), provocaría un cambio en los valores de los participantes que formaban parte del grupo experimental (los que recibían la intervención). En este sentido, los resultados obtenidos permiten confirmar la hipótesis: los participantes del grupo experimental 
obtuvieron un incremento estadísticamente significativo en los valores de universalismo, benevolencia, colectivismo y autotrascendencia en comparación con los participantes del grupo control. Es decir, la práctica de la Meditación Fluir produjo una mejora significativa de los valores de carácter social y altruista, en contraposición a valores más individualistas y egoístas. Así, los participantes del grupo experimental aumentaron sus valores de "comprensión, aprecio, tolerancia y protección para el bienestar de todas las personas y para la naturaleza" (Universalismo), y la "preocupación por preservar y reforzar el bienestar de personas con las que se tiene contacto personal frecuente" (Benevolencia). Asimismo, puntuaron más alto en valores que sirven a intereses colectivistas (intereses de algún colectivo o grupo social: la combinación de valores de Conformidad, Tradición y Benevolencia), y en la dimensión de Autotrascendencia (que incluye Universalismo y Benevolencia), es decir, la preferencia nuevamente por valores que benefician a la colectividad frente a los que favorecen al individuo.

Este incremento en valores que conllevan una preocupación por los demás, valores que trascienden a uno mismo, puede ser debido a que la práctica de la meditación permite a la mente alcanzar el pleno discernimiento, y, de este modo, permite liberarse de los deseos y la ambiciones del ego, al tomar conciencia de que son transitorios, insatisfactorios y ajenos a nosotros mismos (Goleman, 2006). De este modo, cesa la búsqueda permanente de satisfacciones externas que, paradójicamente, nos hace permanecer en un estado constante de insatisfacción que no nos permite disfrutar de lo que somos y de lo que tenemos en cada instante. La sociedad actual nos persuade de que cuantas más experiencias agradables podamos alcanzar, y cuanto más rápidamente pasemos de unas a otras, más felices y realizados seremos. De esta forma, se desarrolla el hábito de desear continuamente, y este hábito del deseo se autoperpetúa al generar apego por algo que se encuentra en otro lugar, hacíendonos desarrollar una sensación de carencia, de que siempre nos falta algo, y de que somos seres incompletos. Al practicar meditación, se toma conciencia de que el deseo es transitorio y carece de esencia, pues tan sólo es un pensamiento que se manifiesta para alejarnos del momento presente y nos sumerge en la esfera de la fantasía, la ilusión y la imaginación (Goldstein y Kornfield, 2002). De este modo, al tomar conciencia del flujo de pensamientos, de su carácter transitorio, al ser capaces de observarlos sin identificarnos con ellos y sin dejarnos arrastrar por su contenido asumiendo que no tienen porque reflejar la realidad, vamos alcanzado una gran sensación de libertad, de dominio, de responsabilidad y de autocontrol. 
Asimismo, con la práctica de la meditación se alcanza una comprensión directa de que cada acto que vaya encaminado a perjudicar a otra persona, al primero que daña y perjudica es a uno mismo, y cada acto dirigido a ayudar a otra persona, al primero que ayuda y beneficia es a uno mismo. Esto es lo que los budistas llaman Dharma, la ley universal. Es por ello, que se produce una disminución en la preocupación por conseguir metas personales e individuales, al tiempo que se incrementa la preocupación por el prójimo y su bienestar.

Finalmente, consideramos que aunque los resultados del presente estudio han sido bastante positivos, hay que ser cautelosos respecto a su generalización por dos razones fundamentales. En primer lugar, porque la muestra de la investigación fue relativamente pequeña. En segundo lugar, porque estuvo formada por personas voluntarias, con los sesgos que esto conlleva. Así mismo, es necesario confirmar la permanencia a lo largo del tiempo de los resultados obtenidos mediante la realización de medidas de seguimiento que confirmen si el cambio obtenido en los valores en el grupo experimental se mantiene posteriormente.

\section{Referencias}

Barnes, V.A., Bauza, L.B. y Treiber, F.A. (2003). Impact of stress reduction on negative school behavior in adolescents. Health Qual Life Outcomes, 1(10), 5-30.

Beaucheim, J. Hutchins, T. y Patterson, F. (2008). Mindfulness meditation may lessen anxiety, promote social skills, and improve academic performance among adolescents with learning disabilities. Complementary Health Practice Review, 13(1), 34-35.

Campagne, D.M. (2004). Teoría y fisiología de la meditación. Cuadernos de Medicina Psicosomática, y Psiquiatría de Enlace, 69/70, 15-30.

Carlin, P. y Lee, K. (1997). Treat the body, health the mind. Health, 11(1), 72-78.

Cohen, J. (1988). Statistical power analysis for the behavioral sciences. Erlbaum: Hillsdale.

Cuadrado, I. (2006). Psicología Social de los valores humanos. En A. Gómez, E. Gaviria e I. Fernández (Coords.), Psicología Social, (pp. 583-617). Madrid: Sanz y Torres.

De la Fuente, J., Peralta, F.J. y Sánchez, M.D. (2006). Valores sociopersonales y problemas de convivencia en la Educación Secundaria. Electronical Journal of Research in Educational Psychology, 4(2), 171-200.

Deshimaru, T. (2006). La práctica del zen. Barcelona: RBA

Franco, C. (2007). Técnicas de relajación y desarrollo personal. Granada: Cepa. 
Franco, C. (2009a). Reducción de la percepción del estrés en estudiantes de Magisterio meditante la práctica de la Meditación Fluir. Apuntes de Psicología, 27, 99-109.

Franco, C. (2009b). Meditación Fluir para serenar el cuerpo y la mente. Madrid: Bubok.

Goldstein, J. y Kornfield, J. (2002). Vipassana. El camino de la meditación interior. Barcelona: Kairós.

Goleman, D. (2006). La meditación y los estados superiores de conciencia. Málaga: Sirio.

González, F. (1990). Educación en valores y diseño curricular. Madrid: Alhambra Longman.

Hart, W. (1994). La Vippasana. El arte de la meditación. Madrid: Luz de Oriente.

Hayes, S. C., Strosahl, K. D. y Wilson, K. G. (1999). Acceptance and Commitment Therapy: An experiential approach to behavior change. New York: Guilford Press.

Hernández, P. (1997). Evaluación de valores y moldes cognitivo-afectivos en la educación socio-afectiva. En A. Cordero (Comp.), La evaluación psicológica en el año 2000 (pp. 149-172). Madrid: Pirámide.

Hernández, P. (2000). Enseñanza de valores socioafectivos en un escenario constructivista: bienestar subjetivo e inteligencia intrapersonal. En J. Beltrán y otros (Coords.), Intervención psicopedagógica y currículum escolar (pp. 217- 254). Madrid: Pirámide.

Hernández, P. (2002). Los moldes de la mente. Más allá de la inteligencia emocional. La Laguna: Tafor.

Hernández, P. y Aciego de Mendoza, R. (1990). Programa Instruccional-Emotivo para el crecimiento y la Autorrealización personal: Aprendiendo a realizarse (PIECAP). (2 edición revisada y ampliada, 1999). Madrid: TEA.

Holen A. y Halvor, E. (2007). Acem Meditation: An introductory companion. Oslo: Acem International.

Iriarte, C., Alonso-Gancedo, N. y Sobrino, A. (2006). Relaciones entre el desarrollo emocional y moral a tener en cuenta en el ámbito educativo: propuesta de un programa de intervención. Electronical Journal of Research in Educational Psychology, 4(1), 177 212.

Jedrczak, A., Beresford, M. y Clements, G. (1985). The TM-Sidhi programme, pure consciousness, creativity and intelligence. The Journal of Creative Behavior 19, 270 275.

Kabat-Zinn, J. (2007). La práctica de la atención plena. Barcelona: Kairós.

Krishnamurti, (1979). La verdad y la realidad. Barcelona: Edhasa.

Lager, E. J. (2007). Mindfulness. La conciencia plena. Barcelona: Paidós. 
Martín-Asuero, A. y García de la Banda, G. (2007). Las ventajas de estar presente: desarrollando una conciencia plena para reducir el malestar psicológico. International Journal of Clinical and Health Psychology, 7(2), 369-384.

Rockeach, M. (1973). The Nature of Human Values. New York: Free Press.

Ros, M. y Grad, H. (1991). El significado del valor trabajo como relacionado a la experiencia ocupacional. Una comparación de profesores de EGB y estudiantes del CAP. Revista de Psicología Social, 6, 181-208.

Schwartz, S.H. (1992). Universals in the content and structure of values: Theoretical advances and empirical test in 20 countries. En M.P. Zanna (ed.), Advances in Experimental Social Psychology (vol.25, pp. 1-65). Nueva York: Academic Press.

Schwartz, S.H. (2001). ¿Existen aspectos universales en la estructura y contenido de los valores humanos? En M. Ros y V.V. Gouveia (Coords.), Psicología Social de los valores humanos. Desarrollos teóricos, metodológicos y aplicados (pp. 53-77). Madrid: Biblioteca Nueva.

Schwartz, S.H. y Bilsky, W. (1990). Toward a theory of the universal content and structure of values: Extensions and cross-cultural replications. Journal of Personality and Social Psychology, 58, 878-891.

Segal, Z.V., Williams, J.M. y Teasdale, J.D. (2006). Terapia cognitiva de la depresión basada en la consciencia plena. Un nuevo abordaje para la prevención de recaídas. Bilbao: Desclée de Brouwer.

Solberg, E.E., Berglund, K.A., Engen, L.E. y Loeb, M. (1996). The effect of meditation on shooting performance. British Journal of Sports Medicine, 30, 1-5.

Trumbulls, M.J. y Norris, H. (1982).Effects of Transcendental Meditation on selfidentity indices and personality. British Journal of Psychology 73, 57 - 68.

Vallejo, M.A. (2006). Mindfulnes. Papeles del Psicólogo, 27(2), 92-99.

Westlund, P. (1993). Acem Meditation beneficial for stressed locomotive engineers. Dyade, 2, 36-51.

Wilson, K. G. y Luciano Soriano, M. C. (2002). Terapia de Aceptación y Compromiso (ACT): Un tratamiento conductual orientado a los valores. Madrid: Pirámide. 